\title{
The Comparison of Cognitive Behavior Therapy with Psychopharmacological Intervention for Women with Anxiety Disorders (GAD, SAD \& OCD)
}

\author{
Abdollah Alizadeh $^{1 *}$, Gayane Shahverdyan ${ }^{2}$, Ahmad Etemadi $^{3}$ \\ ${ }^{1}$ Yerevan State University, Yerevan, Armenia \\ ${ }^{2}$ Faculty of Philosophy and Psychology, Yerevan State University, Yerevan, Armenia \\ ${ }^{3}$ Faculty of Psychology Education Sciences, Allameh Tabatabaei University, Tehran, Iran \\ Email: "aa1347@live.com
}

Received May 22 ${ }^{\text {nd }}$, 2012; revised July 5 ${ }^{\text {th }}$, 2012; accepted August $4^{\text {th }}, 2012$

\begin{abstract}
The first aim of the present research is to determine the effectiveness of Cognitive Behavior Therapy (CBT) in comparison to psychopharmacological intervention on Iranian women with Anxiety disorders. The second aim of the research is to find the relationship between the irrational beliefs and maladaptive thinking with anxiety disorder and the third aim of the research is to investigate which method of treatment causes more satisfaction in the patient. In regard to the research issue, the sample included 300 women with the average age of 18 to 45 who were selected randomly of those patients with anxiety disorders (general anxiety disorder GAD, obsessive compulsive disorder OCD, social anxiety disorder SAD) who referred to counseling and mental health centers and private offices in Eslamshahr city (Tehran state). They were divided into 6 groups: 1) The first three groups with one of these three disorders (GAD, OCD and SAD) referred to Cognitive Behavior Therapy voluntarily; 2) The other three groups with the same disorders referred to the psychiatrist for the psychopharmacological intervention voluntarily. The psychopharmacological intervention course was at least 6 months and the total period of CBT was 12 to 16 sessions (held every week). The diagnosis interview was performed on the basis of DSM-IV-TR 2000 before the treatment. Then the check-list SCL90-R test was performed after diagnosis by the psychologist and in the next session the Jones' irrational beliefs test (IBT) was performed. Research method is of the quasi-experimental method with pre-test and post-test. Generally, the present study findings verify the positive effect of the cognitive-behavior therapy on the change of irrational beliefs, maladaptive thinking and the decrease of symptoms and in the end the research points out that CBT causes more satisfaction than the other treatment in patients; while the psychopharmacological intervention just decreases the symptoms of the disorders, less than CBT. At the end of treatments, both of groups answered to the satisfaction of treatment questioner. It showed that patients were more satisfied with CBT than psychopharmacological intervention. The result of the research showed that CBT not only decreased the symptoms of anxiety disorders but also changed the patients' irrational beliefs, maladaptive thinking and thinking methods. It should be mentioned that such a phenomenon is accompanied by attaining new social skills and in the end, the effect of CBT will be more permanent and stable and patients are not caught in a kind of recovery-relapse cycle.
\end{abstract}

Keywords: Cognitive Behavior Therapy; Psychopharmacological Intervention; Anxiety Disorders

\section{Introduction}

Anxiety disorder is a blanket term covering several different forms of abnormal and pathological fear and anxiety. Conditions now considered anxiety disorders only came under the aegis of psychiatry at the end of the 19th century. Gelder, Mayou \& Geddes (2005) explain that anxiety disorders are classified in two groups: continuous symptoms and episodic symptoms.

Current psychiatric diagnostic criteria recognize a wide variety of anxiety disorders. Recent surveys have found that as many as $18 \%$ of Americans may be affected by one or more of them.

The term anxiety covers four aspects of experiences an individual may have: mental apprehension, physical tension, physic-

${ }^{*}$ Corresponding author. cal symptoms and dissociative anxiety. Anxiety disorder is divided into generalized anxiety disorder, phobic disorder, and panic disorder; each has its own characteristics and symptoms and they require different treatment (Gelder et al., 2005). The emotions present in anxiety disorders range from simple nervousness to bouts of terror (Barker, 2003). Anxiety disorders are among the most common psychiatric disorders, occurring in 5\% - $18 \%$ of all children and adults (Costello, 1995). Anxiety disorders are often associated with impairment in academic, social and personal functioning (Pine, 1997). Significant comorbidity including depression (Kovacs, 1989), substance abuse (Kushner, 1990) and subsequent adult anxiety, major depression and suicide attempts, highlight the need for effective and readily accessible treatments. However, the evidence base for treatment of anxiety in women is relatively limited. The initial trials of cognitive-behavioral therapy (CBT) (Kendall, 1994; Barrett 
1996; Kendall, 1997) were positive (Kendall, 1997). The aim of CBT is to help the women to identify possible cognitive deficits and distortions, to reality-test them, and then to teach new skills or challenge irrational thoughts and beliefs, and replace them with more rational thinking (Kindal, 1990). More specifically, CBT is a psychological model that involves helping the women to: 1) recognize anxious feelings and bodily or somatic reactions to anxiety; 2) clarify thoughts or cognitions in anxiety-provoking situations (i.e. unrealistic or negative attributions and expectations); 3) develop coping skills (i.e. modifying anxious selftalk into coping self-talk); and 4) evaluate outcomes. The behavioral training strategies include: modeling, reality exposure (in-vivo exposure), role-playing and relaxation training. The behavioral treatment is based upon the premise that fear or anxiety are learnt responses (classically conditioned) that can be "unlearnt”.

CBT identifies habitual ways in which patients distort information (e.g., automatic thoughts) and teaches patients to identify and respond to their dysfunctional thoughts and beliefs, using a variety of techniques to change thinking, mood, and behavior.

Research Support, one of the main reasons CBT has become so popular is because of how much research has demonstrated its effectiveness. There are a large number of well-constructed experiments that have shown it to be highly useful in treating depression and anxiety disorders, including GAD. The key factors for it to be helpful are buying in to the belief that it will help, completing relevant assignments and a willingness to confront uncomfortable thoughts, although many CBT techniques can be difficult to do at First, for most people the remission of GAD is well worth the struggle.

\section{The Aim of Research}

The first aim of the present research is to determine the effectiveness of Cognitive Behavior Therapy (CBT) in compareson to psychopharmacological intervention on Iranian women with Anxiety disorders. The second aim of the research is to find out the relationship between the irrational beliefs and maladaptive thinking with anxiety disorder and the third aim of the research is to investigate which method of treatment causes more satisfaction in the patients.

\section{Object of Research}

The object of research is Iranian women with anxiety disorders, especially GAD, ASD \& OCD who referred to mental health center, and private office to be treated with CBT and the psychopharmacological intervention. The total number of the sample is 300 women: 150 women with OCD, GAD and SAD who received CBT and the other 150 women with OCD, GAD and SAD who received psychopharmacological intervention. The individuals' age range is $18-45$.

\section{Hypotheses}

1) Irrational beliefs and maladaptive thinking have a central role in developing anxiety disorders; we must change them into rational beliefs and adaptive thinking or decrease them in order to treat anxiety disorders in a better way. It is just possible by using cognitive-behavior therapy (CBT) because it decreases irrational beliefs more than psychopharmacological intervene- tions.

2) While the psychopharmacological interventions attend to the physiological aspects of symptoms of GAD, SAD \& OCD, CBT directs to change maladaptive thinking patterns and teach social skills and it makes patients more satisfied and ultimately decreases anxiety disorders symptoms more than psychopharmacological interventions.

3) As the symptoms of anxiety disorders through CBT decrease, the comorbidity depression diminishes too. This fact proves that there is an inner connection between depression and anxiety.

\section{Method of Intervention}

The psychopharmacological intervention course was at least 6 months and the total period of CBT was 12 to 16 sessions (held every week).The diagnosis interview was performed on the basis of DSM-IV-TR 2000 before the treatment. Then the check-list SCL90-R test was performed after diagnosis by the psychologist and in the next session the Jones'irrational beliefs test (IBT) was performed. In the end, the satisfaction of treatment inventory was performed on the sample of the research.

\section{Research Instruments}

As it is mentioned, two tests and an inventory were used, Jones' irrational beliefs test (IBT) which assessed irrational beliefs and thoughts and the checklist SCL90-R which used to assess the patients' mental health.

The SCL-90 is one of the most used tests in diagnosing psychological problems. It is a self-report questionnaire originally oriented towards symptomatic behavior of psychiatric outpatients (Derogatis et al., 1973) and it is reviewed on the clinical experiment of Psychometric analysis and its final form were provided in 1976. Validity and reliability of both tests have been approved in the intended society by some researchers many times.

\section{Treatment Satisfaction Inventory}

This is a kind of inventory that has been made by researcher. It consists of 10 questions with 5 parts that pays attention to different aspects of both treatments and measures the patients' attitudes toward them. In the following part, the aspects of questionnaire are being offered.

1) Feeling of satisfaction of gaining the goals;

2) Feeling of satisfaction of the treatment process;

3) Selectivity of the treatment to disorder;

4) Being active during treatment;

5) Feeling of satisfaction of the factors;

6) The fear of treatment;

7) The patient's attitude toward the therapist's devotion of enough time to his problem;

8) Introducing the treatment to others by the patient;

9) Negative attitude toward the treatment aspects;

10) Fear of social limitations in the future.

Method of the scoring of treatment satisfaction inventory:

This questionnaire consists of ten questions in which each question has 5 scales based on Likert 5-degree scale, one the testees (subjects) check off. The total score of it lies in the range of 10 to 50; in some of questions, low scores suggest the more satisfaction and the low concern; while in some of them, high scores show the more satisfaction of the subjects (testes). 
For example in the following question, the high score (5) is the sign of more satisfaction.

1) Have you achieved what you expected of the treatment as we are getting to the end of its course now?

Completely (5) Very much (4) partly (3) No (2) Not at all (1)

\section{The Scientific Innovation}

1) It is the first time that the combination of CBT \& REBT is used in the treatment of anxiety disorders (SAD, GAD \& OCD) in comparison to psychopharmacological intervention in Iranian society.

2) Providing a comprehensive and complete treatment pattern with eight dimensions (Figure 1) that could be used by the other therapists and cause a kind of confidence in patients and professionals that a complete treatment has been done and possibility of relapse will reach to the least level.

3) Offering a satisfaction questionnaire of treatment and measuring the amount of patients 'satisfaction, confidence and interest to the treatment method.

4) The superiority of CBT over psychopharmacological intervention in decreasing both the irrational beliefs and symptoms of anxiety disorders.

5) The illustration of the inner relationship of anxiety disorders and depression, in this way that by decreasing the anxiety symptoms, the comorbidity depression diminishes.

\section{Result}

The data analysis method, in order to answer the questions of research and conclude, the SPSS program has been used for analysis in the two following discusses;

1) Descriptive statistics (mean-standard deviation);

2) Inferential statistics Analysis of Co-Variance (ANCOVA).

The investigation of hypotheses

Before the presentation of the results of covariance analysis, the adjusted means of the treatment groups and disorders have been represented in the Table 1.

The outcome results of the comparing of the mean of the dependent variable (irrational beliefs posttest) in the interaction of the treatment groups with the groups of patients with anxiety disorders have been presented in the following table (Table 2).

The first row of the above table shows that as a result of the appropriate matching of treatment groups, the pretest of the irrational beliefs in the two treatment groups of each disorder is the same and with 95\% confidence there is no significant difference between the pretests, that is expectable $\left(\mathrm{F}_{(1293)}=.22, P\right.$ $>0 / 05$ ). The second row of the Table 2 shows that there is no significant difference between the posttest scores of three groups of patients with anxiety disorder after the adjustment of the pretest effect, and with 95\% confidence the adjusted posttests of the irrational beliefs in three groups of 150 patients is the same $\left.\mathrm{F}_{(1293)}=.33, P>.05\right)$. In contrast, the third row shows that generally there is a significant difference between the mean of the posttest scores of two groups of treatments (each group of 150 patients) and with $99 \%$ confidence the mean of the posttest scores of the irrational beliefs in the psychopharmacological group is greater than the CBT $\left(\mathrm{F}_{(1293)}=1411.28, P<\right.$ $0 / 01)$.

In response to the first hypothesis, the forth row of the Table 2 shows that there is a significant difference (.05) between the mean of the adjusted posttests in the interaction of the treatment groups with anxiety disorders. Regarding the adjusted means in Table 1, the amount of means of three groups of anxiety disor-

Table 1.

The adjusted means of the irrational beliefs posttest.

\begin{tabular}{ccccc}
\hline \multirow{2}{*}{$\begin{array}{c}\text { Anxiety } \\
\text { disorders }\end{array}$} & \multicolumn{2}{c}{ CBT } & \multicolumn{2}{c}{ Psychopharmacological intervention } \\
\cline { 2 - 5 } & Mean & SD & Mean & SD \\
\hline GAD & 202.2 & 3.93 & 327.9 & 3.93 \\
SAD & 208.6 & 3.94 & 333.3 & 3.94 \\
OCD & 208.8 & 3.93 & 337.7 & 3.93 \\
\hline
\end{tabular}

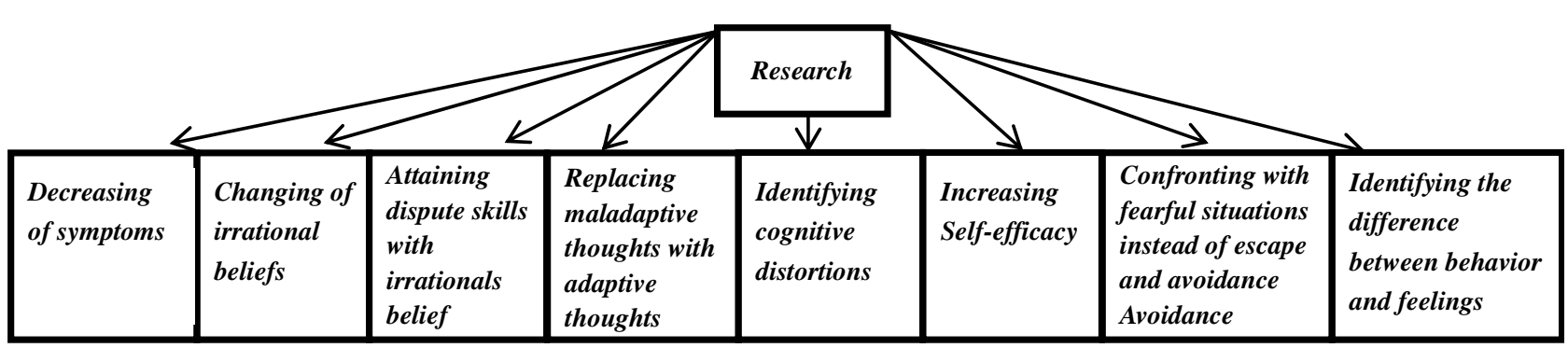

Figure 1.

A comprehensive and complete treatment pattern with eight dimensions.

Table 2.

The results of covariance analysis for the posttest of the irrational beliefs after the adjustment of the pretest effect.

\begin{tabular}{lllllll}
\hline Source of changes & df & SS & MS & Statistic (f) & Sig & Effect size Eta $^{2}$ \\
\hline The irrational beliefs pretest & 1 & 171.26 & 171.26 & .22 & .64 & .001 \\
Anxiety disorders & 2 & 508.87 & 254.44 & .33 & .72 & .002 \\
Treatment groups & 1 & 1087881.12 & 1087881.12 & 1411.28 & .00 & .83 \\
Anxiety disorders treatment groups & 2 & 6299.84 & 3149.92 & 4.09 & .02 & .03 \\
Error & 293 & 225866.39 & 770.87 & & & \\
Total & 300 & $2.364 \times 107$ & & & & \\
\hline
\end{tabular}


ders (each group of 50 patients) that participated in CBT is lower than the amount of means of the other three groups (each group of 50 patients) which participated in psychopharmacological intervention. This shows that the CBT is more effecttive than psychopharmacological intervention in decreasing the irrational beliefs of the patients with anxiety disorders, as the observed statistics of crucial measure in 0.05 level with degree of freedom $=1293$ is greater $\left(\mathrm{F}_{(1293)}=4.09, P<0 / 05\right)$. In the following Figure 2 the adjusted means for three groups of anxiety disorder and for two methods of treatment have been illustrated.

The outcome results of the comparison of the mean values of the dependent variable (disorder symptoms posttest) in the interaction of treatment groups with the groups of patients with anxiety disorders have been presented in the following table (Table 3).

The first row of the table represents that as the result of the appropriate matching of the treatment groups, the pretest of the disorder symptoms in two treatment groups is the same and with $95 \%$ confidence there is no significant difference between the pretests which is expectable $\left(\mathrm{F}_{(1293)}=3.73, P>0 / 05\right)$. According to the second row of the Table 3 , it is observable that there is no significant difference between the posttest scores of the three groups of patients with anxiety disorder after adjusting the pretest effect and with $95 \%$ confidence the adjusted posttests of the disorder symptoms in three groups of 100 anxiety patients is the same $\left(\mathrm{F}_{(1293)}=.81, P>0 / 05\right)$. In Contrast, it is observable through the third row of the table that there is a significant difference between the mean of posttest of two groups of treatment (each group of 150 patients) and with $99 \%$ confidence the mean of adjusted posttest scores of disorder symptoms in psychopharmacological group is greater than that of CBT $\left(\mathrm{F}_{(1293)}=384.62, P<0 / 01\right)$ In response to the second hypothesis, the forth row of the Table 3 shows the significant difference in level 0.01 between the adjusted means of the posttests in the treatment groups' interaction with anxiety disorders. With regard to the adjusted means of the Table 3, it is observable that the means of the disorder symptoms in three groups (each group $=50$ patients) of anxiety disorders that participated in CBT is lower than the means of three other groups



Figure 2.

The adjusted means for three groups of anxiety disorder and for two methods.

Table 3.

The results of the covariance analysis for posttest of the disorder symptoms after the adjustment of the pretest effects.

\begin{tabular}{lllllll}
\hline Effect size Eta $^{2}$ & Sig & Statistic (F) & MS & SS & df & Source of changes \\
\hline .02 & .06 & 3.73 & 265.01 & 265.01 & 1 & The symptom of disorders pretest \\
.01 & .45 & .81 & 31.86 & 63.73 & 2 & Anxiety disorders \\
.57 & .00 & 384.62 & 15151.16 & 15151.16 & 1 & Treatment groups \\
.07 & .00 & 11.52 & 454 & 907.99 & 2 & Anxiety disorders treatment groups \\
& & 39.39 & 11541.96 & 293 & Error \\
& & 265.01 & 2800027.85 & 300 & Total \\
\hline
\end{tabular}


(each group $=50$ patients) that participated in the psychopharmacological intervention. This position represents that the CBT is more effective in the treatment of anxiety disorders than the psychopharmacological intervention. Thus with the $99 \%$ confidence, the observed statistics is greater than the crucial value with 1 and 293 degree of freedom $\left(F_{(1293)}=11.52, P<0 / 01\right)$. In the following diagram (Figure 3 ) the adjusted means of the disorder symptoms for three groups of anxiety disorder and two groups of treatment has been shown.

The results of the covariance analysis and adjusted means in Table 4, represents that CBT decreases all of the subscales of the symptoms of the disorders in patients with anxiety disorder more than the psychopharmacological intervention. But decrease of depression symptom is the aim of the hypothesis (3). The result of the investigation shows that the mean of the cognitive-behavior therapy (.84) in 0.01 level is lower than the

Estimated Marginal Means of postest GSI

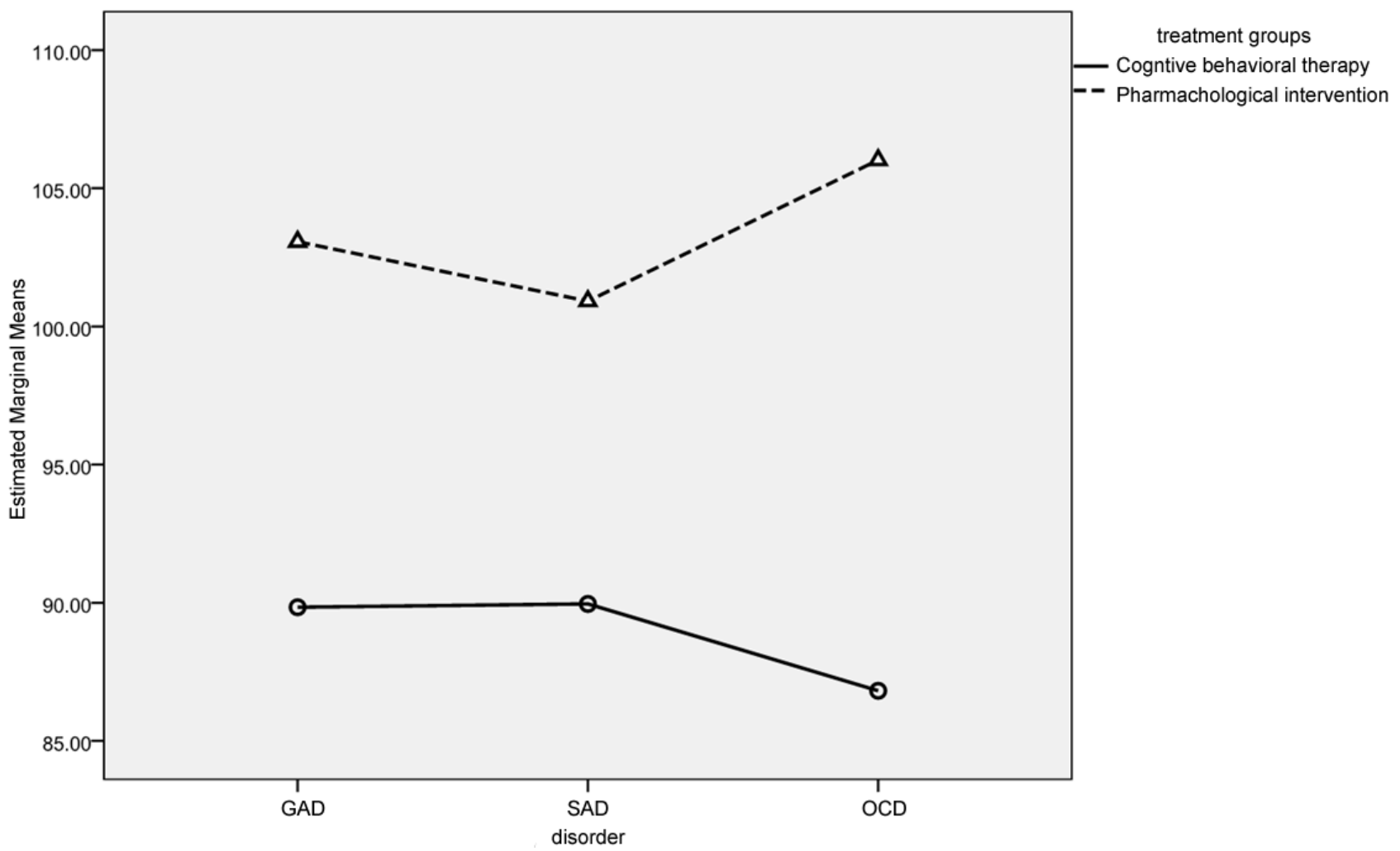

Figure 3.

The adjusted means of disorder symptoms for two groups of treatment and for three groups of patients with anxiety disorder.

Table 4.

The adjusted mean, standard deviation and the result of covariance analysis of subscales and indexes of disorder for anxiety disorders.

\begin{tabular}{|c|c|c|c|c|c|c|}
\hline \multirow{2}{*}{ Effect of Size } & \multirow{2}{*}{ Statistic } & \multicolumn{2}{|c|}{ Psychopharmacological Intervention } & \multicolumn{2}{|c|}{ CBT } & \multirow[b]{2}{*}{ SCL 90-R, Subscales } \\
\hline & & $\mathrm{SD}$ & M & SD & M & \\
\hline Eta2 & $(97 \& 1) \mathrm{F}$ & & & & & \\
\hline .22 & $8.85^{* *}$ & .02 & .85 & .02 & .64 & Somatization \\
\hline .05 & $16.71^{* *}$ & .02 & 1.12 & .02 & .98 & Obsessive (compulsive questions) ${ }^{*}$ \\
\hline .09 & $28.83^{* *}$ & .02 & 1.21 & .02 & 1.07 & Interpersonal sensitivity \\
\hline .05 & $13.22^{* *}$ & .02 & .95 & .02 & .84 & Depression \\
\hline .17 & $61.66^{* *}$ & .03 & 1.69 & .03 & 1.35 & Hostility/aggression \\
\hline .02 & $6.44^{*}$ & .02 & 1.4 & .02 & 1.31 & Phobic \\
\hline .20 & $76.32^{* *}$ & .03 & 1.67 & .03 & 1.36 & Paranoid ideation \\
\hline .11 & $36.07^{* *}$ & .01 & .97 & .01 & .84 & Psychoticism \\
\hline .00 & 71. & .02 & 1.09 & .02 & 1.06 & Additional Questions \\
\hline .55 & $360.13^{* *}$ & .53 & 103.3 & .53 & 88.87 & GSI \\
\hline .40 & $197.53^{* *}$ & .01 & 1.52 & .01 & 1.30 & PSDI \\
\hline
\end{tabular}


mean of the psychopharmacological intervention so that the observed statistics (5.68) is greater than the crucial value $\mathrm{F}_{(1297)}$ $=5.68, P<0 / 05)$. Therefore, with $99 \%$ confidence the symptoms of comorbidity depression in patients with anxiety disorders with CBT decrease.

With regard to the adjusted means of Table 4, it is obvious that the means of the all subscales especially the anxiety symptoms, paranoid thoughts and depression in the posttest of the cognitive-behavior therapy is significantly lower than those of the psychopharmacological Intervention and the researcher's hypothesis areaccepted. According to the obtained results, the researcher's three hypotheses are approved.

\section{Discussion}

As the Tables 1 and 2 and the Figure 2 show, CBT decreases the irrational beliefs and has a significant effect on them, but the psychopharmacological intervention hasn't had any effects on decreasing the irrational beliefs. What is the importance of the decrease of the irrational beliefs in anxious patients? To answer this question, we refer to Albert Ellis' point of view.

Albert Ellis states that anxiety and emotional disorders are caused by the irrational beliefs and illogical thinking. In his opinion, emotions and thoughts are not different and separate reactions. It could be mentioned in this way that as far as the irrational beliefs exist, the emotional disorders remain as usual. Individuals continue up the disorders and irrational behavior by retelling them to themselves. In Ellis' point of view, when an individual becomes captive of irrational thoughts, probably he puts himself in kind of feeling like anger, resistance, defense, guilty, anxiety, numbness, fatigue, lack of control and helplessness. All the people's emotional problems are caused by their magical and superstitious thinking that are not experimentally valid.

When an individual's mental structure usually becomes full of the irrational beliefs, his emotions also are effected by those beliefs and finally the individual's behaviors become irrational, too. Therefore, the first step is to reform our beliefs so that our emotions don't become effected by them. Awareness of the beliefs, dominance on emotions, controlling and managing them in different situations could immunize us against the irrational beliefs. The other appropriate way to overcome these beliefs and prevent life conflicts and quarrels is to replace the irrational beliefs with the rational beliefs (Albert, 1992). In this research, CBT was used to help the patients to change their thinking method through dispute methods which are common in CBT and REBT (Socrates method, asking and changing their self-talk and etc.). In this way, they can challenge their maladaptive thinking methods and the irrational beliefs. In this part, other researchers' findings related to this research have been mentioned to confirm the findings. One research has been done in psychiatric institute in Iran (Mehryar et al., 2000) and its issue was "comparing the effectiveness of the CBT and the psychopharmacological intervention in treating the generalized anxiety disorder". The research findings showed that the CBT is effective in decreasing anxiety, thoughts and unhealthy attitudes and improving the patients' interpersonal relationship. But the psychopharmacological intervention (Benzodiazepines and tricycle anti-depression) has just been effective in decreasing anxiety level. This survey showed that the interpersonal relationships and cognition distortions in patients who received CBT improved more than the other group which received drugs.
The other research has been done on the students of Allameh University in Iran which was supposed to investigate the relationship between their irrational beliefs and mental health. The used instruments in this research were SCL90-R, IBT. The result showed that there is a relationship between the irrational beliefs and mental health and we can anticipate students' mental health through their irrational beliefs (Houshang, Peyman, \& Sepideh, 2002).

The other research investigated the relationship between maladaptive thinking and the suicidal ideation in wives with injured feelings and found that there is a significant relationship between them. This finding is in accordance with the result of the findings of Lester and Golding's research (Keslo et al., 1989, 1998).

Another research which was conducted in this field surveyed the high rate of the irrational beliefs in patients who attempted suicide through self-burning in Tehran and it found that these women's irrational beliefs were higher than the normal people (Kamal, 2000)

Tables $\mathbf{3}$ and $\mathbf{5}$ and Figure 3, represent that the symptoms of disorders which were measured by SCL-90 R, have decreased in CBT more than the other group which was treated by the psychopharmacological intervention. This research and previous researchers' findings confirm that the decrease of the symptoms of disorder will not be permanent and resistant and the possibility of relapse goes up in those who just get the psychopharmacological intervention. Therefore, the patients get into some kind of relapse and recovery circle and after some time patients develop a kind of helplessness that in Martin Seligman' opinion is the cause of depression (learned helplessness).

The present research findings showed that CBT causes more changes in patients' psychological aspects and attitude in comparison to the psychopharmacological intervention.

The gained changes as the result of CBT in patients remained more resistant after the intervention in all aspects of treatment satisfaction. These findings are in accordance with other researches' findings with the same issue. Kat and his colleagues showed that drug therapy for high blood pressure is not just sufficient to improve the patients' life quality and the use of psychological approaches is necessary. The reason to such claim is that most patients with major hypertension don't have the clear illness symptoms and long-term and even life-long taking of anti-hypertensive drugs such as Atenolol, Cilazapril, Verapamil and Propranolol in a long time makes the patients' attitude to them negative. Therefore, drug therapy just has a positive effect on decreasing hypertension and could not change patients' negative attitude to their own illness and take its ill role; but CBT could have a positive effect on improving the patients' life quality along the drug therapy. One of the things that predict the success of the treatment and decrease the severity and negative effects of the illness is to implement the therapeutic recommendations and it could be only achieved through CBT.

Table 5.

The adjusted means of posttest scores for disorder symptoms.

\begin{tabular}{ccccc}
\hline \multirow{2}{*}{ Anxiety disorders } & \multicolumn{2}{c}{ CBT } & \multicolumn{2}{c}{ Psychopharmacological in. } \\
\cline { 2 - 5 } & Mean & SD & Mean & SD \\
\hline GAD & 89.83 & .89 & 103.1 & .92 \\
SAD & 89.97 & .89 & 100.9 & .89 \\
OCD & 86.81 & .90 & 106.02 & .89 \\
\hline
\end{tabular}




\section{Conclusion}

The result of this research emphasizes on the importance of the role of irrational beliefs and maladaptive thinking in individuals' development to anxiety disorders. It points out that disputing the irrational beliefs, maladaptive thinking and cognitive distortion by CBT and REBT which the patients learn during the treatment can be changed into an individual skill, and individuals by using this skill can confront the provokinganxiety situations easily.

A complete and comprehensive treatment is one that beyond decreasing the symptoms of the disorder includes the mentioned eight elements of the research model. Except this, it is possible that a patient gets into a recovery and relapse process. As the results of the researches show, the effect of psychopharmacological intervention is not as stable as the effect of CBT. Most of the patients who are treated just by drugs become involved in relapse again; at last this issue possibly leads the patients to a learned helplessness.

Depression is usually accompanied by anxiety disorders, and vice versa. The three studied disorders in this research are highly accompanied by depression, and anxiety and depression have an inter relationship. It could be said that there is a relationship between these disorders. According to Dr. Johan Rosqvist, if anxiety disorders are not treated, they finally could lead into depression disorders. In other words, the ultimate outcome of anxiety is depression.

Considering this point that the irrational beliefs and maladaptive thinking are the main cores of anxiety disorders, it is necessary to teach people how to confront with these kinds of attitudes in a comprehensive way. It is a kind of prevention that is not only effective but also economical.

Briefly, the research findings point out that through training, individuals can prevent the development of anxiety disorders and depression that generally originate from the irrational beliefs and maladaptive thinking. It is also possible in other places such as schools, colleges, etc.

\section{REFERENCES}

Ajzen, I., \& Fishbein, M. (1977). Attitude-behavior relations: A theoretical analysis and review of empirical research. Psychological Bulletin, 84, 888-914. doi:10.1037/0033-2909.84.5.888

Ajzen, I. (1985). From intentions to actions: A theory of planned behavior. In J. Kuhl, \& J. Beckman (Eds.), Action-control: From cognition to behavior (pp. 11-39). Heidelberg: Springer.

Ajzen, I. (1988). Attitudes personality and behavior. Chicago, IL: Dor- sey.

Alford, B. A., \& Lantka, A. L. (2000). Processes of clinical change and resistance. Behaviour Modification, 24, 566-579. doi:10.1177/0145445500244005

Allen, D. M. (2012). Use of between-session homework in systemsoriented individual psychotherapy. Journal of Psychotherapy Integration, 16, 238-253. doi:10.1037/1053-0479.16.2.238

Ascher, L., \& Efrem, J. (1978). Use of paradoxical intention in a behavioral program for sleep onset insomnia. Journal of Consulting and Clinical Psychology, 46, 547-550. doi:10.1037/0022-006X.46.3.547

Azarmidokht, R. et al. (2011). The relationship between dysfunctional thoughts and suicidal tendencies in spouse abused. Journal of Research and Science Women Society, 3, 123-136.

David, D. (2009). Rational and irrational beliefs: Research, theory, and clinical practice. Oxford: Oxford University Press, 25-26.

Efstratopoulos, A. D., Voyaki, S. M., Baltas, A. A., Vratsistas, F. A., Kirlas, D. E., Kontoyannis, J. T. et al. (2006). Prevalence, awareness, treatment and control of hypertension in Hellas, Greece. American Journal of Hypertension, 19, 53-56. doi:10.1016/j.amjhyper.2005.07.011

Griez, E. J. L., Faravelli, C., Nutt D., \& Zohar, D. (2001). Anxiety disorders. Hoboken, NJ: John Wiley \& Sons Ltd., 10-18.

Hossine, S. (1996). The relationships between mothers' irrational beliefs with their children's behavioral problems. Journal of Applied Psychology, 3, 45-56.

Houshang, G., Peyman, K. Z., \& Sepideh, J. (2002). Assessment between mental health and irrational beliefs. Tehran: Healthy University, 1-20.

Jaleh, A. B., Houshang, M., Taghi, B. M., \& Afsar, A. (2000). Comparative Effectiveness of Drug Therapy Group therapy in the treatment of generalized anxiety disorder. Tehran: Tehran Psychiatric Institute, 125-136.

Kamal, S. (1994). Comparison of Irrational Beliefs in Suicide Attempt (self-burning) with the normal people. Share Kord University Medical Science, 2, 52-60.

Kazantzis, N. et al. (1973). Using homework assignments in cognitive behavior therapy. New York: Routledge, 57-137.

Mehdi, E. et al. (2009). Comparative efficacy of cognitive- behavioral therapy, exposure and response prevention and improve patient Fluoxetine obsessive - compulsive disorder. Tehran: Shahed University, 39-50.

Noyes Jr., R., \& Hoehn-Saric, R. (2006). The anxiety disorders. Cambridge: Cambridge University Press, 3-29.

Rosqvist, J. (2005). Exposure treatments for anxiety disorders a practitioner's guide to concepts' methods, and evidence-based practice. New York: Taylor \& Francis Group, 1-123.

Stoudeh-Asl, N. (2010). Comparison two methods of CBT and medical treatment on hypertension illness life aspect. Medical Journal of Semnan University, 11, 294-302.

Vanin, J. R., \& Helsley, J. D. (2008). Anxiety disorders: A pocket guide for primary care. New York: Humana Press. 